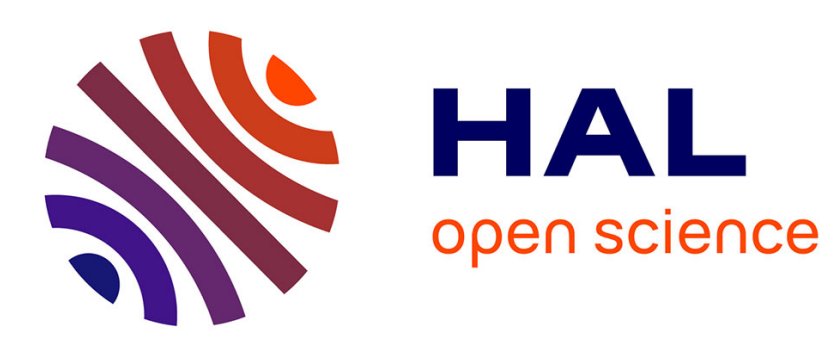

\title{
Systematic Analysis of Active Clock Deskewing Systems Using Control Theory
}

Vinil Varghese, Tom Chen, Peter M. Young

\section{To cite this version:}

Vinil Varghese, Tom Chen, Peter M. Young. Systematic Analysis of Active Clock Deskewing Systems Using Control Theory. DATE'05, Mar 2005, Munich, Germany. pp.820-825. hal-00181216

\section{HAL Id: hal-00181216 https://hal.science/hal-00181216}

Submitted on 23 Oct 2007

HAL is a multi-disciplinary open access archive for the deposit and dissemination of scientific research documents, whether they are published or not. The documents may come from teaching and research institutions in France or abroad, or from public or private research centers.
L'archive ouverte pluridisciplinaire HAL, est destinée au dépôt et à la diffusion de documents scientifiques de niveau recherche, publiés ou non, émanant des établissements d'enseignement et de recherche français ou étrangers, des laboratoires publics ou privés. 


\title{
Systematic Analysis of Active Clock Deskewing Systems Using Control Theory
}

\author{
Vinil Varghese, Tom Chen, Peter M. Young \\ Department of Electrical and Computer Engineering \\ Colorado State University, Fort Collins, CO 80523, USA
}

\begin{abstract}
A formal methodology for the analysis of a closed loop clock distribution and active deskewing network is proposed. In this paper an active clock distribution and deskewing network is modeled as a closed loop feedback system using state space equations. State space analysis allows systematic analysis of any clock distribution and deskewing systems to determine various conditions under which a system can over-compensate and become potentially unstable. Such an analysis can be very useful to designers as they will be able to determine analytically as to how the clock deskewing system behaves. By using the proposed approach, repeated simulations can be greatly limited and maybe entirely avoided. We applied the proposed method to an experimental clock deskewing system to illustrate the effectiveness of the proposed approach. The proposed approach can be further extended to determine performance of such systems under different configurations.
\end{abstract}

\section{INTRODUCTION}

The current level of integration has made clock distribution an increasingly difficult task. The increase in process and environment variations due to aggressive scaling and integration has continued to contribute to increase in clock skew. One effective method of dealing with increasing clock skew in the design process is the adoption of on-chip active skewing system [1].

The existing clock deskewing approaches can be broadly categorized into two different types of designs, namely, open loop and closed loop designs. The open loop design methods for clock distribution are based on implementing geometrically and electrically matched clock distribution structures to minimize skew on the chip. The most commonly used method for distributing clock signals in chips is to insert buffers either at the clock source and/or along a clock path, forming a clock tree structure. This clock source is frequently described as the root of the tree. A mesh version of the clock tree structure is also used in which shunt paths further down the clock distribution network are placed to minimize the interconnect resistance within the clock tree. This mesh structure effectively places the branch resistances in parallel, minimizing the clock skew [2], [3], [4]. Another approach for distributing clock signals utilizes a hierarchy of planar symmetric H-tree or X-tree structures [2], [5], [3], [4]. These methods will no longer be able to achieve the skew requirements in the future due to the complexity of the designs and increased process variations. Several attempts have been made to utilize localized clock skew to improve synchronous performance by providing more time for the critical worst case data paths. However, in order for these methods to be effective, the accurate value of clock skews need to be known during the design. This is difficult to achieve [2], [4], [6], [7]. In order to compensate for skew in real time a closed loop clock distribution system known as the active deskewing system, has been proposed by [8], [9], [1], [10], [11], [12]. Such a system exhibits disturbance rejection and is robust to modeling errors.

For a closed loop feedback system approach employed in an active deskewing scheme, stability is an important criterion. The existing analysis methods for the closed loop deskewing systems are based on repeated brute force simulations. This type of method is very time consuming and does not give a definitive result by which one can say assertively that the system designed is stable or not. Since active deskewing systems employ closed loop techniques, it can be modeled as a closed loop control system using state space equations. This paper describes an analytical approach using the state space equations in control theory. The proposed approach can be used to analyze the stability a given clock deskewing system using the existing control system theories. We will illustrate how the state space equations, both in linear and non-linear models, can be formulated and how some of the existing control system theories can be applied to optimize performance and to determine the stability of a given active deskewing system.

\section{DESCRIPTION OF CLOCK DESKEWING SYSTEM}

In the active clock deskewing system, the entire chip is divided into many clock zones. Figure 1 shows a schematic of a 28-zone clock deskewing system.

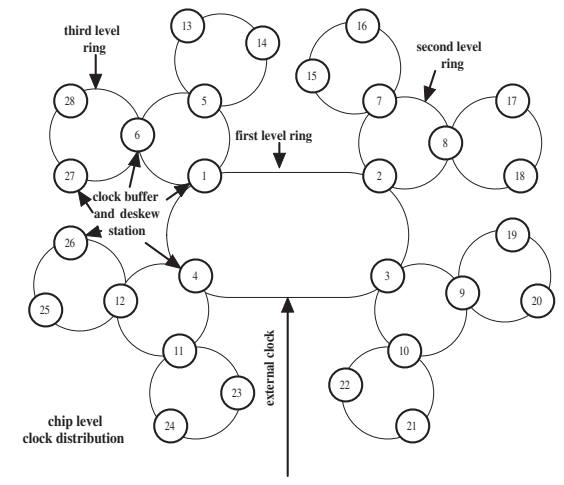

Fig. 1. Block diagram of 28 zone clock distribution network

Clocks in each zone are derived from a clock buffer assigned to that zone. Each of the zones have a certain communication pattern among themselves to ensure that the skew across the chip is within a given specification. The clock buffers in a each zone communicate with each other through a hierarchical ring structure as shown in Figure 1. Each clock buffer adjusts its output based on the phase difference in the arrival time of the clock between it and its neighbors. When skew at a certain spot of the chip is increased, the clock buffers within this region will be automatically adjusted to minimize the skew within this region. This will in turn cause increase in region-to-region skew. Thus, in each of the regions, the skew will be automatically adjusted. Hence a wave of adjustment is carried out throughout the chip until the skew is within the required specification. The logical implementation of the deskew stations is shown in Figure 2. [13] describes this logical implementation in detail. A slightly complex example of the implementation of such a scheme is shown in Figure 3 . The 27-zone system is more complex compared to the 28 -zone system due to the higher level of hierarchy, which makes it more difficult to analyze in terms of performance. 


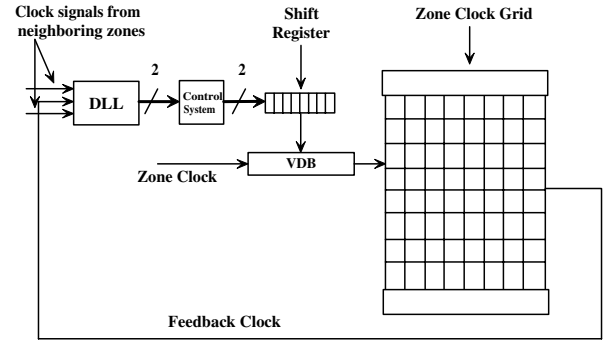

Fig. 2. Logical implementation of a deskew station

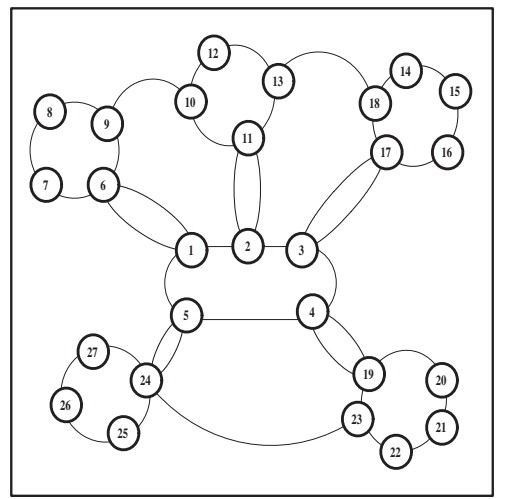

Fig. 3. Block diagram of 27 zone clock distribution network

\section{SYSTEM MODELS USING CONTROL THEORY}

The active clock deskewing scheme is a non-linear discrete timeinvariant MIMO control system. It can be initially modeled as a linear system in order to understand the dynamics of the system better. We will present the linear system representation first, followed by a more accurate representation using non-linear state space equations. The approximation used in the linear system involved using linear gain values in the system matrix, rather than using non-linear elements that saturate the adjustments in the outputs.

The linear model for the active clock deskewing scheme can also be used to analyze stability for optimization of the acquisition time. The acquisition time for the system is defined as the number of clock cycles the system takes to deskew on the occurrence of noise to the system in the form of jitter. The two different linear models, namely, the un-optimized linear model and the optimized linear model, are discussed in the following subsections.

\section{A. Un-optimized Linear Model}

Let $\operatorname{Tarr}_{k}$ be a vector of the arrival times of the clock in all the clock zones at clock cycle k; let $T_{n o m}$ be the nominal clock period from the PLL; and let $U 1$ be a matrix describing the connections among clock zones along with the gains that are associated with each connection. The state space equation for updating (adjusting) clock period at clock cycle $k+1$ is:

$$
\operatorname{Tarr}_{k+1}=T_{n o m}+\operatorname{Tarr}_{k}+U 1 \cdot \operatorname{Tarr}_{k}
$$

The values of gains in the matrix $U 1$ is chosen such that the following equation is satisfied:

$$
|\operatorname{eig}(1+U 1)|<1
$$

If the absolute value of the eigen values of $(1+U 1)$ is less than one, it implies that the system is stable.

Equation (1) can be better explained using a simple case of a clock distribution network employing the proposed deskewing scheme.
Consider an active clock deskewing scheme as shown in Figure 4. This network has 4 clock zones. Assume the arrival times of all the

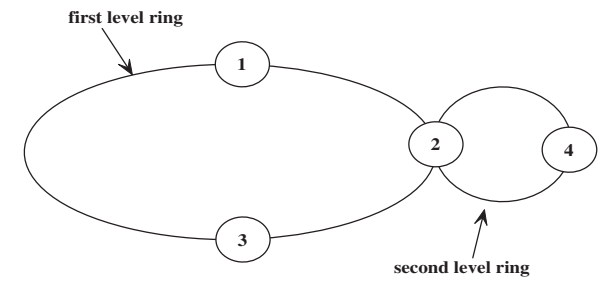

Fig. 4. A simple active deskewing network with 4 clock zones

zones except zone 2 to be zero. The zones compare their current and past arrival times of the clock signal with their neighbors. A jitter of $20 \mathrm{ps}$ is introduced to zone 2 . The matrix $\operatorname{Tarr}_{k}$ can be written as:

$$
\operatorname{Tarr}_{k}=\left[\begin{array}{c}
0 \\
0 \\
20 \\
0 \\
0 \\
0 \\
0 \\
0
\end{array}\right]
$$

Let the nominal arrival time of the system be $350 \mathrm{ps}$, hence the vector $T_{n o m}$ is:

$$
T_{n o m}=\left[\begin{array}{c}
350 \\
350 \\
350 \\
350 \\
350 \\
350 \\
350 \\
350
\end{array}\right]
$$

The matrix $U 1$ can be written as:

$U 1=\left[\begin{array}{cccccccc}-k 1 & -k 1 & 0 & 0 & k 1 & k 1 & 0 & 0 \\ 1 & -1 & 0 & 0 & 0 & 0 & 0 & 0 \\ k 2 & k 2 & -2 * k 2 & -2 * k 2 & 0 & 0 & k 2 & k 2 \\ 0 & 0 & 1 & -1 & 0 & 0 & 0 & 0 \\ 0 & 0 & k 1 & k 1 & -k 1 & -k 1 & 0 & 0 \\ 0 & 0 & 0 & 0 & 1 & -1 & 0 & 0 \\ 0 & 0 & k 1 & k 1 & 0 & 0 & -k 1 & -k 1 \\ 0 & 0 & 0 & 0 & 0 & 0 & 1 & -1\end{array}\right]$

Using the stability criterion mentioned in Equation (2), the values of $\mathrm{k} 1$ and $\mathrm{k} 2$ were found to be 0.06 and 0.07 respectively. The matrix $U 1$ for this example is:

$U 1=\left[\begin{array}{cccccccc}-0.06 & -0.06 & 0 & 0 & 0.06 & 0.06 & 0 & 0 \\ 1 & -1 & 0 & 0 & 0 & 0 & 0 & 0 \\ 0.07 & 0.07 & -0.14 & -0.14 & 0 & 0 & 0.07 & 0.07 \\ 0 & 0 & 1 & -1 & 0 & 0 & 0 & 0 \\ 0 & 0 & 0.06 & 0.06 & -0.06 & -0.06 & 0 & 0 \\ 0 & 0 & 0 & 0 & 1 & -1 & 0 & 0 \\ 0 & 0 & 0.06 & 0.06 & 0 & 0 & -0.06 & -0.06 \\ 0 & 0 & 0 & 0 & 0 & 0 & 1 & -1\end{array}\right]$

The Table I shows the skew between the clock arrival times of all the four zones.

TABLE I

SKEW OF THE CLOCK ZONES FOR 8 CLOCK CYCLES.

\begin{tabular}{lcccccccc}
\hline clk cycle & 1 & 2 & 3 & 4 & 5 & 6 & 7 & 8 \\
\hline \hline Zone 1 & 0 & -1.2 & -3.288 & -4.5 & -4.8 & -4.4 & -3.9 & -3.2 \\
Zone 2 & 20 & 17.2 & 12.004 & 8 & 5.3 & 3.5 & 2.4 & 1.6 \\
Zone 3 & -20 & 16 & -8.716 & -3.5 & -0.5 & 0.9 & 1.5 & 1.6 \\
Zone 4 & -20 & 16 & -10.876 & -3.5 & -0.3 & -0.9 & 1.5 & 1.8 \\
\hline
\end{tabular}

As can be seen from Table I, absolute value of the maximum skew at the first clock cycle was 20 ps and at the end of the eighth clock cycle it reduced to $3.2 \mathrm{ps}$. 


\section{B. Optimized Linear Model}

In closed loop active clock deskewing systems, the acquisition time is a very important factor. The un-optimized clock deskewing scheme can be modified to improve the acquisition time. This was achieved by modeling the entire system as a discrete linear quadratic regulator (DLQR) [14]. The basic state space equation remains the same as that for the un-optimized systems (Equation (1)), with the only difference being that the matrix $U 1$ gets modified. The state space equation for the optimized deskewing system is:

$$
\operatorname{Tarr}_{k+1}=T_{n o m}+\operatorname{Tarr}_{k}+U 2 \cdot \operatorname{Tarr}_{k}
$$

By modeling the linear system as a linear quadratic regulator, the output can be kept small using as little as input as possible. This allows a trade-off to be made between the input effort and the regulation of the output and can be formulated as a least squares problem. The optimization is done using Riccati recursions performed by DLQR.

The matrix $U 2$ was generated using DLQR which generates a discrete linear quadratic regulator. All the inputs to the system except the Matrix $U 1$ remain the same. The matrix $U 1$ is replaced by the matrix $U 2$. The matrix $U 2$ for the example in Figure 4 is:

$U 2=\left[\begin{array}{cccccccc}-0.76 & 0 & 0.24 & 0 & 0.24 & 0 & 0.24 & 0 \\ 0 & -0.76 & 0 & 0.24 & 0 & 0.24 & 0 & 0.24 \\ 0.24 & 0 & -0.76 & 0 & 0.24 & 0 & 0.24 & 0 \\ 0 & 0.24 & 0 & -0.76 & 0 & 0.24 & 0 & 0.24 \\ 0.24 & 0 & 0.24 & 0 & -0.76 & 0 & 0.24 & 0 \\ 0 & 0.24 & 0 & 0.24 & 0 & -0.76 & 0 & 0.24 \\ 0.24 & 0 & 0.24 & 0 & 0.24 & 0 & -0.76 & 0 \\ 0 & 0.24 & 0 & 0.24 & 0 & 0.24 & 0 & -0.76\end{array}\right]$

Table II below shows the skew between the clock arrival times of all the four zones. It is clear that the acquisition time of the optimized linear model is shorter than that of un-optimized linear model.

TABLE II

SKEW OF THE CLOCK ZONES FOR 8 CLOCK CYCLES CALCULATED FROM TABLE??.

\begin{tabular}{lcccccccc}
\hline Clock cycle & 1 & 2 & 3 & 4 & 5 & 6 & 7 & 8 \\
\hline \hline Zone 1 & 0 & 0 & 0 & 0 & 0 & 0 & 0 & 0 \\
Zone 2 & 20 & 0.0099 & -0.0001 & 0 & 0 & 0 & 0 & 0 \\
Zone 3 & -20 & -0.0099 & 0 & 0 & 0 & 0 & 0 & 0 \\
Zone 4 & -20 & -0.005 & 0.0001 & 0 & 0 & 0 & 0 & 0 \\
\hline
\end{tabular}

\section{Non-Linear Model}

The non-linear model of active deskewing systems takes into account some practical issues of noise and implementation. When correcting the clock arrival time for a given zone based on skew, the amount of correction is often discrete due to quantized increments and decrements of delay. To account for quantized adjustments, we define step as the minimum amount of delay by which the variable delay buffer (VDB) increases or decreases the arrival time of the clock at each clock zone. In addition, adjustments to the clock arrival time of a clock zone should be made based on "real" skew between clock zones, rather than false skew caused by potential jitter. Therefore, adjustments to the clock arrival time of a clock zone will be made if there are consecutive skew comparison results of the same direction between two clock zones. To simplify the discussion without losing generality, we assume that the window for preventing clock jitter is 3 clock cycles. That is: a clock zone in the deskewing system needs to wait for consistent "increase" or "decrease" requests from its neighbors in 3 consecutive clock cycles before it will adjust its own clock output. The corrections to a clock zone can be written as:

$$
\begin{aligned}
\Delta \operatorname{Tr}_{1}= & f\left(\operatorname { m o d } ( \operatorname { c l k } k _ { - } \text { cycle } , n ) \cdot \operatorname { s t e p } \cdot f \left(\frac { 1 } { 3 } \left(\operatorname{sign}\left[U \cdot \operatorname{Tarr} 1_{k}\right]\right.\right.\right. \\
& \left.\left.+\operatorname{sign}\left[U \cdot \operatorname{Tarr} 2_{k}\right]+\operatorname{sign}\left[U \cdot \operatorname{Tarr} 3_{k}\right]\right)\right)
\end{aligned}
$$

where $\operatorname{Tarr} 1_{k}$ is the current state vector containing the arrival time of the clock in each zone of the deskewing system at the $k^{\text {th }}$ instant. $\operatorname{Tarr} 2_{k}$ is the state vector containing the arrival time of the clock in each zone of the distribution at the $(k-1)^{t h}$ instant. Tarr $3_{k}$ is the state vector containing the arrival time of the clock in each zone of the distribution at the $(k-2)^{t h}$ instant. These past values of arrival times are used so as to make a correction only if the same type of correction (either positive or negative) was requested in the previous two consecutive clock cycles as well. For example, consider the case when the previous two comparisons of clock arrival times for any zone required the zone to speed up the clock arrival time for that zone. If the current comparison also requires the clock arrival time to speed up, only then will the clock arrival time for that zone be sped up. The function $f$ in Equation (4) is a threshold function and can be defined as:

$$
\begin{aligned}
f(x) & =1, x \geq 1 \\
& =0,-1>x<1 \\
& =-1, x \leq-1
\end{aligned}
$$

The term $f\left(\frac{1}{3}\left(\operatorname{sign}\left[U \cdot \operatorname{Tarr} 1_{k}\right]+\operatorname{sign}\left[U \cdot \operatorname{Tarr} 2_{k}\right]+\operatorname{sign}[U \cdot\right.\right.$ $\left.\left.\operatorname{Tarr} 3_{k}\right]\right)$ ) checks if the values of the past two comparisons were the same as the result of the current comparison. It outputs a 1 or a -1 depending on whether three consecutive comparisons required the delay to be increased or decreased by a step size, and 0 otherwise.

The function, $\bmod \left(c_{k} k_{-} c y c l e, n\right)$, in Equation (4) allows the adjustments to the clock arrival time of clock zones n-1 clock cycles out of every $n$ clock cycles. The one remaining clock cycle in every $\mathrm{n}$ clock cycles are used for the global clock arrival time adjustment to accommodate the centering of arrival time. This may be desirable to ensure that there is no systematic drift of clock arrival time at the global level. Such a scheme can be modeled as:

$\Delta \operatorname{Tr}_{2}=5 \cdot$ step $\cdot\left[1-f\left(\bmod \left(\operatorname{clk} k_{-} c y c l e, n\right)\right)\right] \cdot f\left(\operatorname{period} \cdot k-\operatorname{Tarr} 1_{k}\right)$

Equation (6) describes a mechanism that speeds up or slows down the clock in each zone once in every $\mathrm{n}$ clock cycles by five times the nominal step size where period $\cdot k$ represents the nominal clock arrival time at time $\mathrm{k}$.

The nonlinear model to describe the active deskewing system can be formulated as:

$$
\operatorname{Tarr} 1_{k+1}=\operatorname{Tarr} 1_{k}+\Delta \operatorname{Tr}_{1}+\Delta \operatorname{Tr}_{2}
$$

\section{STABility ANALYSis USING CONTROL TheORY}

The stability analysis for the linear models can be simply performed by observing the absolute value of the eigen values of the system matrix as shown in Equation (2).

Analytically proving stability of a nonlinear time-invariant system is very difficult in general. However, if the nonlinearity of the system fits into a certain set of special cases, a variety of methods can be used for stability analysis [15],[16],[17]. One of the unifying methods for nonlinear control system stability analysis is integral quadratic constraints (IPC) [18],[19],[20],[21],[22]. In the IQC framework, the nonlinear functions can be bounded using sector-bounded limiting nonlinearity. In other words, the nonlinear functions in a system are replaced by linear functions that are upper bound of the nonlinear functions. We then prove whether the control system with the linear upper-bound functions is stable under any circumstances. Stability analysis for linear systems is well established and understood. If the sector-bound linear system is proven to be stable, the nonlinear system must also be stable. However, if the sector-bound linear system can not be proven to be stable, the stability of the nonlinear system is unknown. 
In the system model in Equation (7), the nonlinear functions mod, sign, and the threshold function $f$ are bounded using linear functions. One of the sector blocks (sector-bound linear functions) used in the IQC analysis toolbox [21] is shown in Figure 5. For example, the

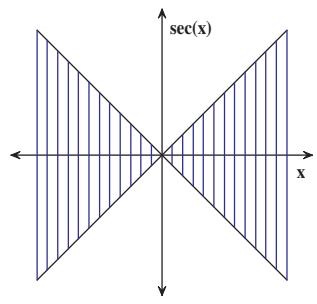

Fig. 5. Sector block in IQC toolbox

nonlinear element $f$ in Equation (7) can be approximated using a modified sector block:

$$
\frac{1}{2}+\frac{1}{2} \cdot \sec
$$

This modified sector block is shown in Figure 6. The function $f$

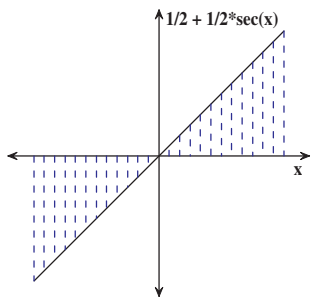

Fig. 6. Modified sector function

can be approximated using the modified sector block as shown in Figure 7 . The nonlinear element $f\left(\bmod \left(c l k_{-} c y c l e, 10\right)\right)$ can be

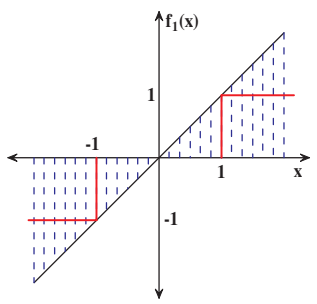

Fig. 7. Approximation of element $f$ using the modified sector block

approximated using the modified sector in Equation (8) as shown in Figure 8. Approximation of $\left[1-f\left(\bmod \left(\operatorname{clk}_{-} c y c l e, 10\right)\right)\right]$ is shown in Figure 9.

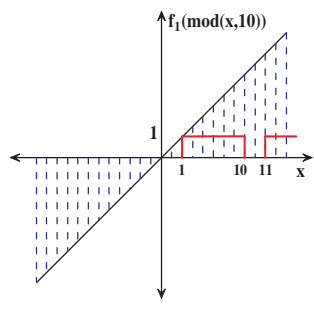

Fig. 8. Approximation of element $f\left(\bmod \left(\mathrm{clk}_{-} c y c l e, 10\right)\right)$ using the modified sector block

Using these modified sector blocks, the entire nonlinear system model can be built in SIMULINK using the IQC toolbox. Equation 9

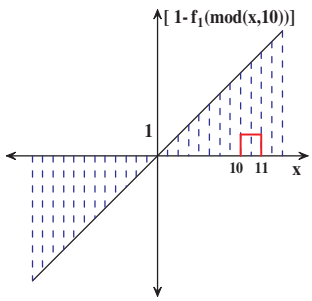

Fig. 9. Approximation of element $\left[1-f\left(\bmod \left(\operatorname{clk}_{c} y c l e, 10\right)\right)\right]$ using the modified sector block

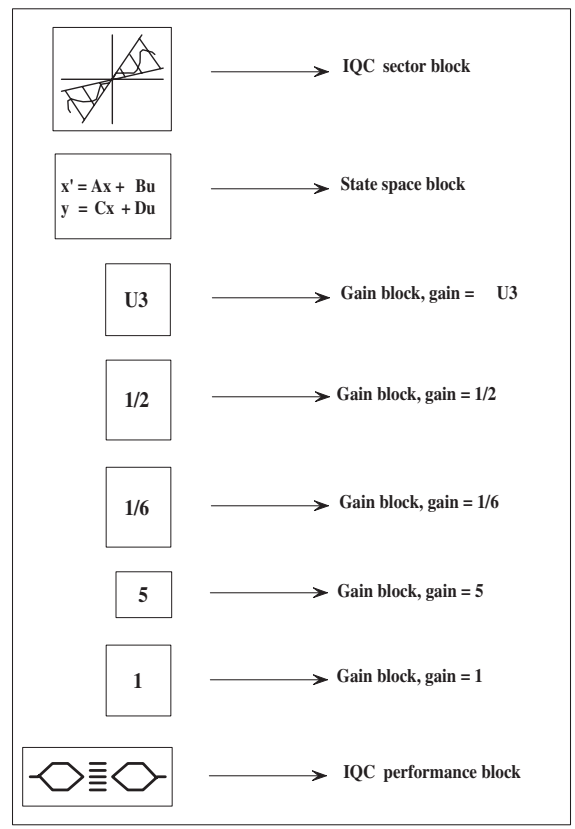

Fig. 10. Blocks used in IQC toolbox

describes the nonlinear model after replacing the nonlinear elements in Equation (7) with sector blocks from the IQC toolbox.

$$
\begin{aligned}
\operatorname{Tarr} 1_{k+1}= & T_{\text {nom }}+\operatorname{Tarr} 1_{k}+\left(\frac{1}{2}+\frac{1}{2} \cdot \mathrm{sec}\right) \cdot \operatorname{step} \cdot\left(\frac{1}{2}+\frac{1}{2} \cdot \mathrm{sec}\right) \\
& \cdot\left(U \cdot \operatorname{Tarr} 1_{k}\right)+\left(\frac{1}{2}+\frac{1}{2} \cdot \mathrm{sec}\right) \cdot\left(U \cdot \operatorname{Tarr} 2_{k}\right) \\
& \left.\left.+\left(\frac{1}{2}+\frac{1}{2} \cdot \mathrm{sec}\right) \cdot\left(U \cdot \operatorname{Tarr} 3_{k}\right)\right)\right) \\
& +5 \cdot \operatorname{step} \cdot \frac{1}{2}+\frac{1}{2} \cdot \mathrm{sec} \cdot \frac{1}{2}+\left(\frac{1}{2} \cdot \mathrm{sec}\right) \\
& \left.- \text { states } 1_{k}\right)
\end{aligned}
$$

To help illustrate the modeling of the proposed non-linear deskewing system, Figure 10 shows the various blocks present in the IQC toolbox that were used to build the simulink model of the nonlinear system. The SIMULINK block diagram of the 27-zone nonlinear active deskewing system for analysis of stability using IQC toolbox is shown in Figure 11.

\section{EXPERIMENTAL RESULTS}

simulations for the linear and non-linear models were performed and all simulations were performed for a duration of 300 clock cycles. External noise in the form of clock jitter was introduced to all test systems at clock cycles $10,60,110,160,210$, and 260 . Figures 12 and 13 show the skew of each of the zones in the 28 -zone system for linear and non-linear models, respectively. The results show that both the linear and the non-linear systems deskew the external clock 


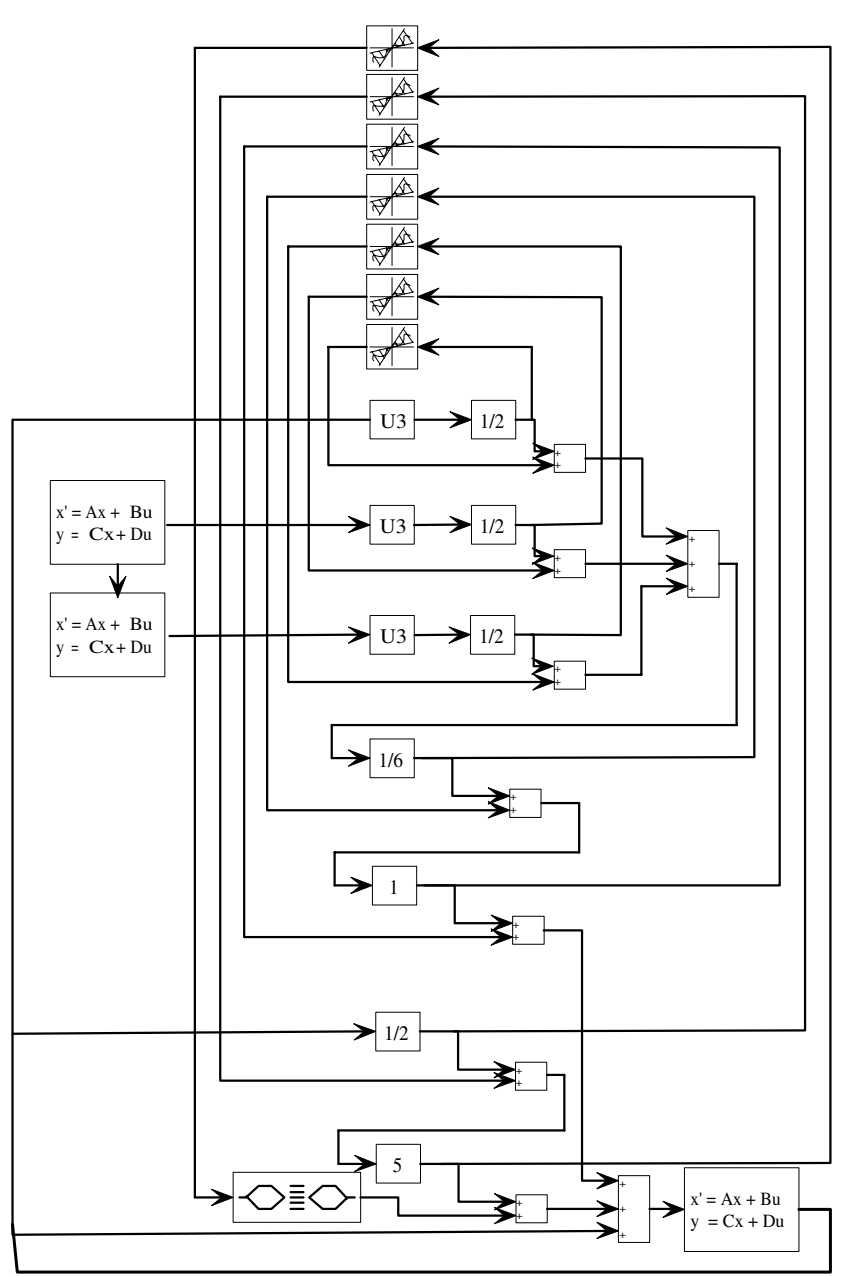

Fig. 11. Block Diagram of the Nonlinear Model using IQC toolbox

disturbances. Both systems exhibit very low residual clock skew (12 ps) after deskewing is completed. Figures 14 and 15 show the skew of each of the zones in the 27-zone system for linear and non-linear models, respectively. The residual skew in the 27 -zone systems is generally lower than those in the 28-zone systems due to the increased complexity in connection patterns.

Stability analysis of the nonlinear model was performed by implementing the modified nonlinear model in Equation (9) where the nonlinear elements were replaced using the sector blocks. The delay blocks were created using state space models since the IQC toolbox does not contain discrete delay elements in the iqc_lib SIMULINK library. The performance block was used to analyze the stability of this nonlinear system. The performance block is required to be used by the iqc_gui command in order to determine if the system is stable or not. The iqc_gui command when used to determine stability of systems, returns an output value of $i q c_{-}$gain if the system is stable else it does not return any value for $i q c_{-}$gain. Step size for the deskewing system was set to $0.5 \mathrm{ps}$.

It is possible that the entire deskewing system becomes unstable. This happens when the clock zones keep over-compensating for the skew. Stability is guaranteed only for a certain range of step size by which the adjustment to the arrival time of the clocks are adjusted. The range for values of step in Equation (9) depends on the topology of clock distribution network. It also depends on how often the adjustments need to be done on the clock arrival times. For the 4-zone system shown in Figure 4, the value of step for which the system

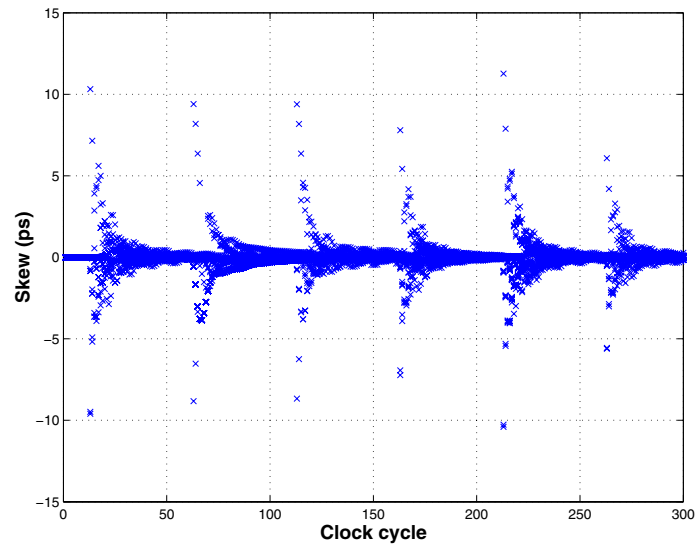

Fig. 12. Clock skew of each zone for the linear un-optimized 28-zone clock distribution system.

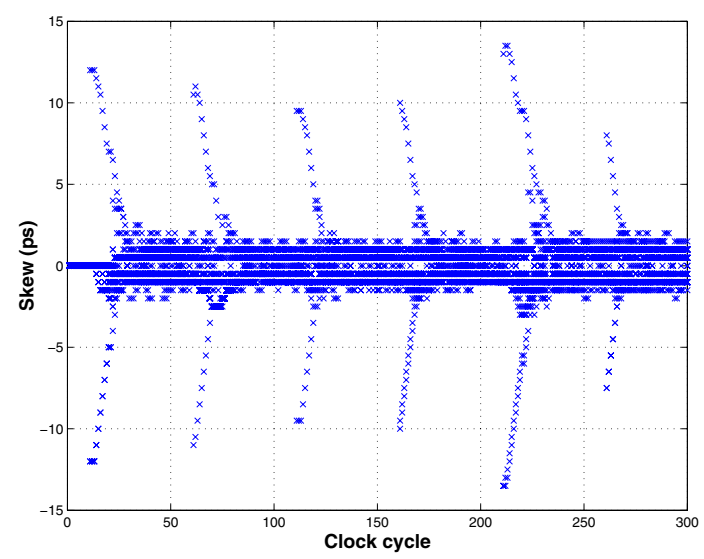

Fig. 13. Clock skew of each zone for the nonlinear 28-zone clock distribution system.

became unstable was found to be 9 ps. The simulation results for the 4-zone system using the nonlinear model in Equation (9) is shown in Figure 16. As seen in the results, the skew of each zone in the systems keeps increasing since the system overcompensated for the skew, thus making the system unstable. This result is also confirmed by using IQC stability analysis.

In general, it is relatively easier for Monte Carlo simulations to detect an unstable system. However, it is not possible for Monte Carlo simulations to prove a system in stable. The confidence grows with increasing amount of simulations. The proposed approach using control theory can prove a system is stable a much less amount of effort.

Simulations for stability analysis for the nonlinear systems were performed using the IQC-toolbox for Matlab. The CPU time for stability analysis for the 28-zone and 27-zone systems were 350 and 276 minutes, respectively, running on a $1.9 \mathrm{GHz}$ Pentium 4 processor with $512 \mathrm{MB}$ of memory.

\section{CONCLUSIONS}

With increasing clock frequencies and higher constraints on clock skew due to increased process and temperature variations, active clock deskewing schemes can be effectively used to reduce clock skew. Current methods for stability analysis of such closed loop active deskewing systems use brute force approaches and involve simulating the systems for very long periods of time with varying set of input conditions and without a definite answer as to whether the 


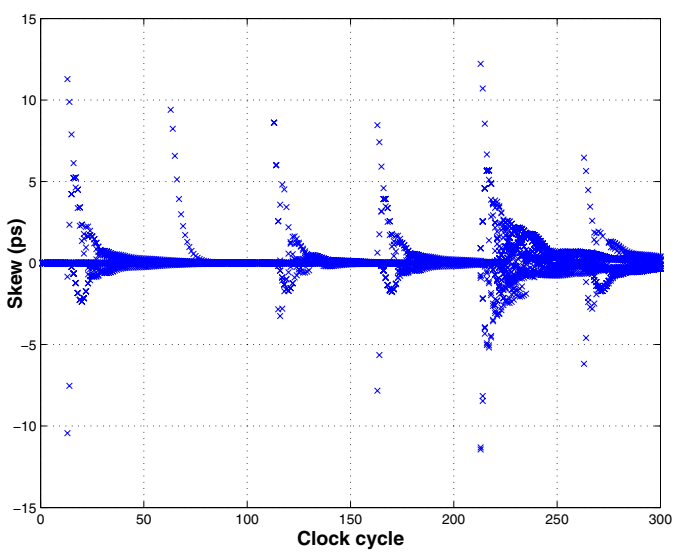

Fig. 14. Clock skew of each zone in an un-optimized 27-zone clock distribution system.

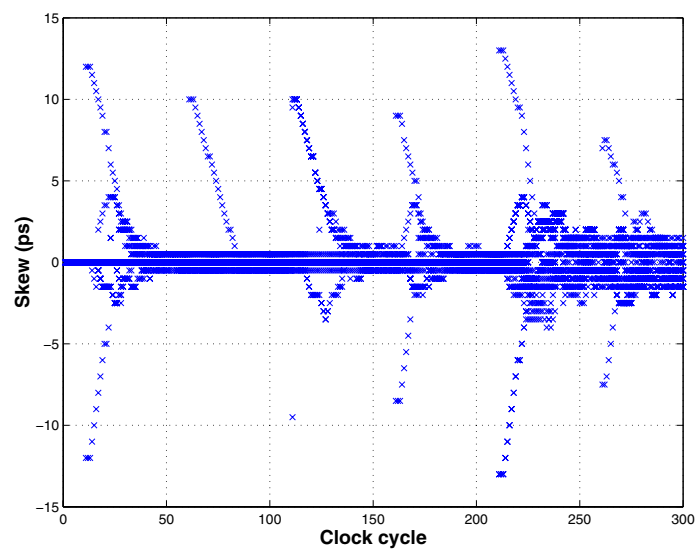

Fig. 15. Clock skew of each zone in non-linear 27-zone clock distribution system.

system is stable or not. This paper presents a systematic approach to analyze the stability of closed loop active deskewing systems using a control system theory approach. Linear models allow faster evaluation of systems, and more importantly, allow experiments on trading off system complexity and acquisition time using DLQR. The non-linear models allow more realistic modeling of the actual systems. However, the computational complexity for stability analysis using IQC increases significantly. Experimental 28-zone and 27-zone active deskewing systems were modeled as a closed loop feedback system both in linear and non-linear approach and analyzed with existing control theory analysis tools to determine its performance and stability. The stability of the systems was demonstrated using the proposed method.

\section{REFERENCES}

[1] S. Rusu, S. Tam, U. N. Desai, R. Kim, and I. Young, "Clock Generation and Distribution for the first IA-64 Microprocessor," Proc IEEE Int. Solid-State Circuits Conf.

[2] E. G. Friedman, "Clock Distribution Networks in Synchronous Digital Integrated Circuits," Proceedings of IEEE, pp. 665-692, May 2001

[3] K. D. Wagner, "Clock system design," IEEE Des. Test Compute., pp. 9 27, Oct. 1998.

[4] E. G. Friedman, "Clock dsitribution design in VLSI circuits- An overview," Proceedings of IEEE Int. Symp. Circuits and Systems, pp. 1475-1478, May 1993.

[5] N. H. E. Weste, Principles of CMOS VLSI Design : A System Perspective. Addison Wesley, 2rd ed., 1993.

[6] M. A. Cirit, "Clock skew elimination in CMOS VLSI," Proceedings of IEEE Int. Symp. Circuits and Systems, pp. 861-864, May 1990.

[7] H. Sutoh and K. Yamakoshi, "A clock distribution technique with an automatic skew compensation circuit," Proc. IEICE Tran. Electron., pp. 277-283, Feb. 1998.

[8] T. W. Chen and P. M. Young, "Internal technical report," Colorado State University, 2002.

[9] S. Rusu and S. Tam, "Clock Generation and Distribution for the first IA64 Microprocessor," Proc. IEEE Int. Solid-State Circuits Conf., pp. 176177, Feb. 2000

[10] G. Geannopoulos and X. Dai, "An adaptive digital deskewing circuit for clock distribution networks," Proc. IEEE Int. Solid-State Circuits Conf., ISSCC'98, pp. 400-401, 1998.

[11] R. L. Aguiar and D. M. Santos, "Highly efficient multi-point clock distribution networks,"

[12] G. Singer and S. Rusu, "The first IA-64 microprocessor: A design for highly parallel execution," ISSCC Dig. Tech. Papers, pp. 422-423, Feb. 2000.

[13] V. Vinil, T. Chen, and P. Young, "Stability analysis of active clock deskewing systems using a control theoretical approach," ASP-DAC 2005, Jan. 2005.

[14] T. Kailath, Linear Systems. Prentice-Hall, Englewood Cliffs, New Jersey, 1980.

[15] G. Balas, J. Doyle, K. Glover, A. Packard, and R. Smith, $\mu$-analysis and synthesis. MUSYN Inc. and The Mathworks Inc., 1991.

[16] C. Desoer and M. Vidyasagar, Feedback System: Input-Output Properties. Academic Press, 1975.

[17] M. Safonov, "Stability margins of diagonally perturbed multivariable feedback systems," IEE Proc., pp. 251-256, 1982.

[18] K. Goh and M. Safonov, "Robust analysis, sectors, and quadratic functionals," IEEE Conf. on Decision and Control, pp. 1988-1993, 1995.

[19] U. Jonsson and A. Rantzer, "Unifying format for multiplier optimization," American Control Conf., pp. 3859-3860, 1995.

[20] A. Rantzer and A. Megretski, "System analysis via integra quadratic constraints," IEEE Conf. on Decision and Control, pp. 3062-3067, 1994.

[21] A. Megretski, C. Y. Kao, U. Jnsson, and A. Rantzer, A guide to IQC $\beta$ : Software for robustness Analysis.

[22] R. M. Kretchmar, P. M. Young, C. W. Anderson, D. C. Hittle, M. L. Anderson, and C. C. Delnero, Robust reinforcement learning control with static and dynamic stability.

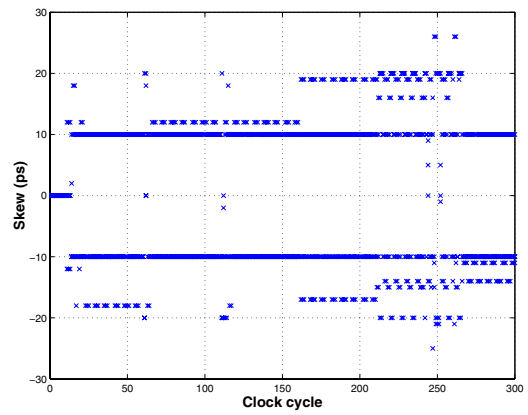

Fig. 16. Clock skew for each zone in an unstable 4-zone system 